\title{
Impacto de las Dificultades de Atención en la Realización de cuidados con Dispositivos Personales - Gafas - en personas con Esquizofrenia.
}

\section{Attention Impairment in the Accomplishment of Cares with Personal Devices - Glasses - in Individuals with Schizophrenia}

\author{
Cardoso, Monica M. ${ }^{\mathrm{i}}$, Molinette, Pedrita ${ }^{\text {ii }}$

\section{RESUMEN}

Los déficits cognitivos, tales como los trastornos de atención, afectan alrededor del $98 \%$ de las personas con esquizofrenia, aspecto muy importante para determinar el nivel de discapacidad. Constituye muchas veces, un factor de mayor disfunción, que los síntomas reales de la propia esquizofrenia. El factor atención es importante por la relación directa que este tiene con la calidad de vida, con las actividades sociales, funcionales y profesionales del individuo. La recuperación no se alcanza solamente en el control de los síntomas, sino preeminentemente con habilitación cognitiva. Considerando que dichos déficits interfieren directamente en la ejecución de actividades que influyen en la autonomía del individuo, se optó por verificar el impacto del déficit de atención en la realización de actividades de vida diaria (AVD): cuidados con los dispositivos personales - gafas. Las actividades de vida diaria se consideran importantes en todos los ámbitos de atención en Terapia Ocupacional; la recuperación de las AVD contribuyen de manera provechosa y significativa para la calidad de vida. Con el propósito de discutir teórica y prácticamente estos aspectos, fue realizado un estudio bibliográfico y una investigación de campo en donde se usaron dos cuestionarios: "Cuidados con las Gafas “(de la autora, 2009) y "Cuestionario de la Atención” [1], aplicados en dos individuos con esquizofrenia que cumplieron con los criterios de inclusión. Los resultados apuntan que aunque los déficits de atención estén presentes en estos individuos, no interfieren de manera significativa en las AVD y no influyen en la ejecución de los cuidados de los dispositivos personales - gafas. Es mas, el $20 \%$ de las personas de esta encuesta deben usar gafas por prescripción médica, aunque desestiman su uso.

PALABRAS-ClAVE: Atención Cognitiva. Esquizofrenia. Actividades de la vida diaria. Dispositivos personales.

\footnotetext{
${ }^{\mathrm{i}}$ Profesora del curso de Terapia Ocupacional del Departamento de Ciencias de la Salud de la Universidad Federal do Paraná, situado em la calle Padre Camargo, 280 - Alto da Glória - CuritibaPR-BR- CEP: 80060-240, teléfono (41) 3360-7273 / (41) 8812-6505, correo electrónico: terapeutamonica@hotmail.com

ii Académica del curso de Terapia Ocupacional del Departamento de Ciencias de la Salud de la Universidad Federal do Paraná, situado em la calle Padre Camargo, 280 - Alto da Glória - CuritibaPR-BR- CEP: 80060-240, teléfono (41) 3360-7273 / (41) 9948-0978, correo electrónico:
} pedritam@hotmail.com 


\begin{abstract}
Cognitive deficits, such as attention disorders, affect about $98 \%$ of individuals with schizophrenia, very important aspect in determining the level of disability. It often represents a major factor of dysfunction than the symptoms of schizophrenia. The ability to pay attention is relevant because of the direct relation with quality of life and social, functional and professional performance of individuals. Recovery is not only achieved by controlling symptoms, but overall with the cognitive recovery. Considering that those deficits directly interfere in the performance of activities that influence in individual's autonomy, it was opted to verify the impact of attention deficit in activities of daily living (ADL) performance; personal devices care - glasses. Activities of Daily Living are considered important in all the modes of health care in Occupational Therapy. The recovery of ADL contributes in a significant and productive way to the quality of life. With the purpose of discussion theory and practice of these aspects, a bibliographic study was done. Furthermore a field research was carried out in which two questionnaires were used: "Care with glasses" (from the author, 2009) and "Attention questionnaire" [1], these were applied in two individuals with schizophrenia that fulfilled the inclusion criteria. Results point that, though attention deficits were present in these individuals, they not interfere significantly in ADL's, and not influence the performance in personal devices care -glasses. Even more, $20 \%$ of the people in this survey must use glasses by medical prescription, though they do not use them.
\end{abstract}

Key words: Cognitive Attention. Schizophrenia. Activities of Daily Living. Personal devices.

\title{
INTRODUCCIÓN:
}

La esquizofrenia es un trastorno mental crónico, que impacta de manera profunda y destructiva a los individuos que la padecen [2] . Según Salgado [2] 98\% de los esquizofrénicos son afectados por alteraciones cognitivas y el $80 \%$ de ellos presentan déficits cognitivos significativos.

Kandel, Schwartz, Jessel [3] afirman que la esquizofrenia es la fragmentación de la mente, en la que los procesos cognitivos son independientes de la voluntad, del comportamiento y de la emoción. Este trastorno presenta también, alteraciones del pensamiento, del autoconocimiento, de la percepción, de los sentimientos, de sus propios actos y de la interacción social. Cerca de $1 \%$ de la población mundial sufre de este trastorno, considerado el más devastador del ser humano, siendo responsable de aproximadamente el 30\% de las hospitalizaciones en el mundo entero [3].

Se caracteriza por delirios y alteraciones de la sensopercepción que desfiguran la realidad, alucinaciones o percepciones distorsionadas (ejemplo: oír voces), lenguaje desorganizado, acciones agitadas o inmovilidad excesiva (comportamiento catatónico), emociones embotadas y pérdida del interés o de la iniciativa, acompañados de una respuesta anormal [4]. Puede ser clasificada según Crow en tipo I con síntomas positivos/productivos que involucra comportamientos tales como alucinaciones y delirio, o tipo II con síntomas negativos/deficitarios que involucra déficits tales como embotamiento afectivo, bloqueos, comportamiento ilógico, falta de higiene, falta de motivación, ausencia de sensaciones de placer y aislamiento social [5]. Sin embrago, según Salgado [2], a pesar de los síntomas positivos y negativos existe también un conjunto de síntomas cognitivos comprometidos, siendo los síntomas positivos y negativos sólo el foco central de la patología. El pronóstico de la esquizofrenia generalmente es negativo ya que implica recaídas del comportamiento psicótico, y tales recaídas van produciendo un deterioro progresivo [3]. 
La literatura da cuenta, que tests y encuestas realizadas para evaluar la existencia de déficits cognitivos en la esquizofrenia, señalan que la atención, la memoria y las funciones ejecutivas son menoscabadas [6]. La cognición se refiere a la capacidad que permite a los individuos desempeñar actividades en las áreas personal, social y/o ocupacional [7]. Por tanto, los déficits cognitivos son factores determinantes en los niveles de discapacidad, siendo mucho más significativos que la propia sintomatología esquizofrénica [8]. Los déficits cognitivos son considerados los mas importantes factores de la disfunción social y ocupacional en los individuos que los padecen [9], y pueden redundar en limitación en la adquisición de nuevas habilidades, pues la autonomía y el funcionamiento social y ocupacional necesitan de estas, para aprender nueva información y así utilizarlas de forma adaptativa [10]. Los déficits cognitivos son importantes pues influyen directamente en la calidad de vida y poseen correlaciones particulares con varias dimensiones de la vida como, por ejemplo, el desempeño en actividades de la vida diaria, actividad laboral, independencia y autonomía de habilidades psicosociales [2] Así, Salgado [2] afirma que la recuperación de los individuos con esquizofrenia no se limita solamente al control de los síntomas, si no también y principalmente, a la mejora cognitiva. Las alteraciones cognitivas pueden ser resultantes de una alteración primaria de la atención [11], pues la "incapacidad de seleccionar la información puede resultar en una desorganización tal, que ninguna actividad podría ser ejecutada" [12].

En cuanto al proceso de atención, este se refiere al mantenimiento del estado de alerta, del control ejecutivo y de la orientación de la entrada sensorial [13] y complementa [14] la conformación de varios procesos cognitivos como la vigilancia, la atención dividida, la memoria y la fluencia verbal. Así Bell, supone que no existen déficits de la atención particulares de la esquizofrenia, pero si deficiencias globales de la atención. La atención, dice, respecto a la percepción selectiva y direccionada a una fuente de información durante determinado tiempo, es una fuerza interna que focaliza la percepción y es factor determinante en los procesos cognitivos y de aprendizaje [15]. Para Gazzani, Ivry, Mangun [16] la atención es un mecanismo cerebral cognitivo que hace posible que informaciones, pensamientos y acciones importantes sean procesados mientras estímulos de menor importancia son ignorados. Los trastornos de la atención son resultado de dificultades en identificar estímulos relevantes y procesarlos, y, en filtrar los estímulos irrelevantes [15].

La atención, a la que nos referimos, es el tipo de atención necesaria para ejecutar la actividad de la vida diaria: el cuidado con dispositivos personales - gafas - se refiere a una atención direccionada por periodos de tiempo prolongados [15]. En este tipo de atención como estado de alerta, se encuentra entre la vigilia y la disposición para la acción cuando existe la necesidad de una respuesta rápida, transformada rápidamente en respuesta a los estímulos externos cuando estos se presentan con carácter de amenaza o si se requiere respuesta rápida, de una actividad para otra [17].

Se entiende así, la importancia de la evaluación de la capacidad de atención en personas con esquizofrenia [18], considerando que las alteraciones de esta son importantes [7] y perjudica el desempeño en las demás habilidades cognitivas [18]. Este es uno de los temas mas estudiados y considerados como más importantes en la psicopatología de la esquizofrenia [19]. Deficiencias de la atención también pueden impactar, acentuando los mecanismos de la patología e influyendo en las funciones más importantes de la vida diaria [7]. Según Kandel et al. [3], los individuos con esquizofrenia - casi en su totalidad - presentan déficits de atención, lo que demuestra la importancia de estudiar este déficit en la ejecución de actividades cotidianas.

Las actividades de la vida diaria son orientadas para que el individuo cuide su propio cuerpo y son imprescindibles para la vida social, lo que permite la supervivencia básica y el bienestar [20]. Según 
la AOTA [20], las actividades de la vida diaria reflejan los valores culturales de las personas y dan significado y estructura a la vida cotidiana, así como atienden a las necesidades del autocuidado, relaciones provechosas y participación social. Transitorios o crónicos, los déficits influyen en la independencia y en la ejecución de actividades [21], como por ejemplo la perdida de la capacidad de cuidar de las propias necesidades y de administrar el ambiente[22], resultando en perjuicios de la autoestima, privacidad personal, ejecución de roles ocupacionales, y otros [21]. El cuidado con los dispositivos personales, que incluye las gafas, es una de las actividades de la vida diaria relevante para la higiene personal y necesaria en la cotidianeidad, lo que puede ser un problema para una persona con esquizofrenia [20].

El ítem cuidado con los dispositivos personales está incluido en las actividades de la vida diaria por la AOTA [20] e incluye: "usar, limpiar y mantener el cuidado de objetos personales, tales como aparatos auriculares, lentes de contacto, gafas, prótesis, equipos de adaptación y contraceptivos sexuales". Sin embargo, para desarrollar este estudio, se optó por analizar el uso de gafas, considerándose que es el dispositivo personal mas comúnmente utilizado entre los adminículos de cuidado personal, además de tener un impacto considerable en la calidad de vida de las personas con deficiencia visual. En enfermedades encronizadas, tal como la esquizofrenia, es importante preservar la calidad de vida, ya que se trata de una enfermedad que requiere tratamiento a largo plazo [23].

Es relevante apuntar que la esquizofrenia provoca un impacto considerable en la calidad de vida y en el funcionamiento psicosocial por poseer síntomas positivos y negativos [24], aún cuando el tratamiento está dirigido hacia toda la sintomatología. Promover la calidad de vida ha sido reconocido como uno de los objetivos terapéuticos de la máxima relevancia [24]. Por otra parte, los esquizofrénicos tienen necesidades extras relacionadas con el estigma y el preconcepto de la patología por presentar limitados recursos personales, tales como habilidades sociales y cognitivas. La constante permanencia en tratamiento y la rutina de medicación psicótica con sus efectos adversos que altera el bienestar y calidad de vida del individuo, resultan en dificultad en la obtención y permanencia laboral [24]. También de observó que la esquizofrenia produce aislamiento social y provoca grandes perjuicios ocupacionales [25].

La oportunidad de realizar actividades significativas y de valor pueden proporcionar a los individuos con trastornos mentales una gran oportunidad para mejorar su calidad de vida, ya que el "hacer", está directamente relacionado con la salud y el bienestar. Tales actividades se refuerzan de manera reciproca y benigna [27]. Por lo tanto, el propósito de este estudio es demostrar la eventual interferencia en la capacidad de atención en individuos con esquizofrenia que pueda dañar la ejecución de actividades de cuidado con los dispositivos personales, gafas.

\section{METODOLOGIA}

El estudio fue realizado con 30 individuos (26 hombres y 4 mujeres) que participaron del CAPS Centro de Atención Psicosocial II - Bigorrilho - y de la Asociación Arnaldo Gilbert, diagnosticados con esquizofrenia por un médico psiquiatra, tomando como referencia, la CIE (Clasificación Internacional de Enfermedades y Problemas Relacionados a la Salud), farmacológicamente estabilizados y sin otro compromiso neurológico asociado.

Los individuos seleccionados para participar de dicho estudio tuvieron acceso al Consentimiento Libre e Informado (TCLI) y a los procedimientos relativos a su participación. 
Fueron seleccionados dos individuos, que cumplían con los criterios de dicho trabajo para responder a la encuesta en sus días de tratamiento en el local.

Se les aplicó el "Cuestionario de la Atención" [1] que contiene 12 preguntas relacionadas a la auto percepción de la atención, con las siguientes alternativas:

- Sin alteraciones o sin problemas como antes;

- Solamente perjudica de vez en cuando;

- Perjudica solo algunas veces;

- Perjudica frecuentemente;

- Perjudica el tiempo entero.

La puntuación es multiplicada de acuerdo con la columna marcada para la obtención del resultado. El puntuación 48 indica grave compromiso de la atención, y la puntuación 0 indica ninguna alteración de la atención.

Enseguida, fue aplicado el cuestionario sobre los "Cuidados con Las Gafas" (elaborado para este estudio), éste contiene 5 preguntas que indagan:

- Frecuencia de la limpieza de las gafas,

- Factor determina tal limpieza.

- Materiales utilizados para la limpieza.

- Criterios de confirmación de la tarea realizada.

- Lugar en que son guardadas.

El resultado es comprobado de acuerdo con la alternativa marcada corroborando si hay o no compromiso de la atención en la realización de cuidados con las gafas.

Las encuestas fueron realizadas dentro de salones con mesas, sillas, iluminación correcta y aislada del ruido externo. Dicha encuesta esta respaldada por el Comité de Ética do Setor de Ciencias da Saúde da UFPR y por el Comité de Ética da Secretaria Municipal de Saúde de Curitiba PR.

\section{RESULTADOS}

\begin{tabular}{|c|c|c|}
\hline & PERSONA 1 & PERSONA 2 \\
\hline SEXO & Masculino & Masculino \\
\hline EDAD & 65 & 38 \\
\hline \multicolumn{3}{|c|}{ CUESTIONÁRIO DE LA ATENCIÓN } \\
\hline PUNTUACIÓN & 10 (compromiso leve) & 30 (compromiso grave) \\
\hline \multicolumn{3}{|c|}{ CUIDADOS COMO LAS GAFAS } \\
\hline R1 & Todo el dia. & Todo el dia. \\
\hline $\mathbf{R 2}$ & Darse cuenta de que están sucios. & Darse cuenta de que están sucios. \\
\hline $\mathbf{R 3}$ & Usa cualquier paño para limpiarlos. & Usa paño especial. \\
\hline $\mathbf{R 4}$ & Comprueba que está limpio. & Comprueba que está limpio. \\
\hline R5 & $\begin{array}{l}\text { A un lugar específico, sin estuche } \\
\text { para gafas. }\end{array}$ & En el estuche para gafas. \\
\hline
\end{tabular}




\section{DISCUSIÓN}

A partir de la aplicación del "Cuestionario de la Atención" (SOHLBER et al., 1994), se constató que los dos individuos presentan déficits de atención y este puede estar relacionado con la patología que padecen. El individuo 1, aunque no presenta compromiso atencional significativo, obtuvo puntuación 10, que demuestra dificultades de la atención, para él, la puntuación que determina la atención en el cuidado de las gafas, equivale a 0 . El individuo 2 tiene puntuación 30 con compromiso más grave de la atención, más arriba que el compromiso medio (24), lo que indica déficit significativo de la atención al aproximarse de la puntuación de compromiso total, que es 48.

El cuestionario "Cuidados con Las Gafas" demostró que ambos individuos, considerando las funciones mentales y las habilidades cognitivas de la atención, limpian las gafas al darse cuenta que están sucias, y al hacerlo, verifican si no quedaron residuos y si están bien limpias, lo que los incluye en contextos y ambientes sociales y culturales.

El uso de las gafas durante todo el día, como lo relatan los dos individuos, puede ser relacionado con un hábito o rutina insertos en los patrones del desempeño ocupacional.

Evaluando los 30 pacientes estudiados, alrededor del $20 \%$ tendría que usar gafas por prescripción médica, y no lo hacen, demostrando la transgresión en sus contextos sociales. No obstante, es posible sugerir que existe un compromiso en la calidad de vida, considerándose que la dificultad visual perturba el desempeño satisfactorio en la realización de actividades de la vida diaria.

Con la finalidad de responder la pregunta central de este estudio, sobre el compromiso de la atención en individuos con esquizofrenia, a partir de la pesquisa realizada con dos individuos que la padecen, se pudo constatar: a) ambos presentan déficit de la atención; b) este déficit no perjudica a estos individuos en la ejecución de cuidados con los dispositivos personales - gafas.

Este estudio también confirma los hallazgos de la literatura encontrada sobre este tema. Dado que el número de individuos estudiados es bajo, proponemos nuevos estudios relacionados al cuidado con otros dispositivos, así como comprobar el motivo de la no adherencia al uso de gafas para la corrección del déficit visual en otros individuos.

El resultado obtenido coincide con la literatura consultada, en cuanto a la presencia de déficits de atención en individuos con esquizofrenia, pero nuestro resultado en dos estudios de caso; requiere aumentar los participantes.

Con la elaboración de dicho estudio, se pudo verificar que el compromiso de la atención no causa impacto en la realización de actividades de la vida diaria, como el cuidado con dispositivos personales - gafas. Además, entre los individuos estudiados, un número significativo no se adscribe a la recomendación médica en torno al uso de gafas. Esta última constatación supone interferencia en la calidad de vida de estos individuos pues los déficits visuales pueden generar perjuicios en la realización de actividades. 


\section{REFERENCIAS BIBLIOGRÁFICAS}

1. SOHBERG, M.M.; JOHNSON, L.; PAULE, L.; RASKIN, S.A.; MATEER, C.A. Attention Process Training II. Puyallup, WA: Association Neuropsychological Research and Development, 1994.

2. SALGADO J. V. Neuropsicologia da Esquizofrenia. En: FUENTES D et al., Neuropsicologia - teoria e prática. São Paulo: Artmed, 2008.

3. KANDEL E. R.; SCHWARTZ J. H.; JESSEL T. M. Princípios da neurociência. 4. ed. São Paulo: Manole, 2003.

4. KOLB B.; WHISHAW I. Q. Neurociência do comportamento. São Paulo: Manole, 2002.

5. KAPLAN V. A.; SADOCK B. J. Compêndio de psiquiatria. 9. ed. São Paulo: Artmed, 2007.

6. KLINGBERG S.; WITTORF S.; WIEDEMANN G. Disorganization and cognitive impairment in schizophrenia: independent symptom dimensions? European Archives of Psychiatry and Clinical Neuroscience de 2006, 256: 532-40.

7. MONTEIRO L. C.; LOUZÃ M. R. Alterações cognitivas na esquizofrenia: conseqüências funcionais e abordagens terapêuticas. Revista Psiquiatria Clínica de 2005, 34 (2). Disponível em: www.scielo.br/pdf/rpc/v34s2/a06v34s2.pdf (acessado em 02/06/2009).

8. BARRERA A. Los transtornos cognitivos de la esquizofrenia. Revista Chile NeuroPsiquiatria de 2006, 44 (3) 06. Disponível em: www.scielo.cl/scielo.php?pid=S07172272006000300007\&script=sci_arttext (acessado em: 22/05/2009).

9. BELL M.; BRYSON G.; GREIG T.; CORCORAN C.; WEXLER B. E. Neurocognitive enhancement therapy with work therapy: effects on neuropsychological test performance. American Medical Association of General Psychiatry de 2001, 58 (8). Disponível em www.bases.bireme.br/cgi-bin/wxislind.exe/iah/cys (acessado em 25/05/2009).

10. SHARMA; ANTONOVA, 2003

11. KAIRALLA I. C. J.; VIEIRA M.A.; MATTOS P.; SHIRAKAWA I. Atenção e esquizofrenia. Psychiatry Brazil de 1999, 4. Disponível em www.polbr.med.br/arquivo/atencao.htm (acessado em 30/05/2009).

12. LURIA A.R. Curso de Psicologia Geral Atenção e Memória. Rio de Janeiro: CIV Bras., 1979, 3: $1-38$.

13. BELL M. D.; FISZDON J. M.; BRYSON G. Attention training in schizophrenia: Differing responses to similar tasks. Journal of Psychiatric Research de 2009, 43: 490-496.

14. Klingberg, Wittorf, Wiedemann (2006) cit. [6]

15. NABAS T R, XAVIER G F. Atenção. In: ANDRADE V M, SANTOS F M, BUENO O F A. Neuropsicologia hoje. São Paulo: Artes Médicas, 2004.

16. GAZZANIGA M. S; IVRY R. B.; MANGUN G. R. Neurociência cognitiva: a biologia da mente, 2. ed. São Paulo: Artmed, 2006.

17. GRIEVE J. Neuropsicologia em Terapia Ocupacional - exame da percepção e cognição. 2. ed. São Paulo: Santos, 2006.

18. SMID J.; NITRINI R. Avaliação Neurológica. In: NETO M R L et al. Psiquiatria básica, 2. ed. São Paulo: Artmed, 2007.

19. SIEDMAN et al., 1983).

20. American occupational therapy association (AOTA). Occupational Therapy practice framework: domain \& process. 2. ed., 2008.

21. MELLO M. A. F.; MANCINI M. C. Métodos e técnicas de avaliação nas áreas de desempenho ocupacional. In: CAVALCANTI A.; GALVÃO C. Terapia Ocupacional: Fundamentação \& Prática. Rio de Janeiro: Guanabara Koogan, 2007.

22. FOTI, 2005, p. 132 
23. SOUZA L. A. S.; COUTINHO E. S. F. Fatores associados à qualidade de vida de pacientes com esquizofrenia. Revista Brasileira de Psiquiatria de 2006, 28 (1).

24. WEHMEIER P. M.; KLUGE, M.; SCHNEIDER, E., SCHACHT, A.; WAGNER, T.; SCHREIBER, W. Quality of life and subjective well-being during treatment with antipsychotics in out-patients with schizophrenia. Progress in Neuro-Psychopharmacology \& Biological Psychiatry de 2007, 31 (703-712).

25. CARDOSO C. S.; CAIAFFA, W.T.; BANDEIRA, M.; SIQUEIRA, A.L.; ABREU, M.N.S.; FONSECA, J.O.P. Qualidade de vida e dimensão ocupacional na esquizofrenia: uma comparação por sexo. Cadernos de Saúde Pública de 2006, 22.

26. SEMKOVSKA, M.; BÉDARD, M.A.; GODBOUT, L.; LIMOGE, F.; STIP, E. Assessment of executive dysfunction during activities of daily living in schizophrenia. Progress in NeuroPsychopharmacology \& Biological Psychiatry, v. 31, p. 289-300, 2004.

27. EKLUND M. Work status, daily activities and quality of life among people with severe mental illness. Quality of Life Research de 2009, 18: 163-170.

28. Organização Mundial da Saúde - OMS (2003), Classificação Estatística Internacional de Doenças e Problemas Relacionados à Saúde - CID-10. $9^{a}$ revisão, São Paulo: Editora da Universidade de São Paulo. 